\title{
COMPARAÇÃO DA TTTT® (TIBIAL TUBEROSITY TRANSPOSITION TOOL) COM A TÉCNICA TRADICIONAL DE TRANSPOSIÇÃO E FIXAÇÃO DA TUBEROSIDADE TIBIAL EM CÃES COM LUXAÇÃO PATELAR: REVISÃO BIBLIOGRÁFICA
}

\author{
COMPARISON OF TTTT® (TIBIAL TUBEROSITY TRANSPOSITION TOOL) WITH THE \\ TRADITIONAL TECHNIQUE FOR TIBIAL TUBEROSITY TRANSPOSITION AND FIXATION IN \\ DOGS WITH PATELLAR LUXATION: LITERATURE REVIEW
}

\author{
C. L. CONSTANTIN ${ }^{1}$, L. H. GAIGA ${ }^{2}$, M. P. FERREIRA ${ }^{3}$
}

\begin{abstract}
RESUMO
A luxação de patela representa casuística importante entre as afecções ortopédicas que acometem o membro pélvico dos cães. É consenso que a luxação de patela é sinal clínico e não donça, sendo causada por deformidades musculoesqueléticas, como a torção externa ou interna da tíbia proximal. A transposição da tuberosidade tibial (TTT) é técnica frequentemente empregada para correção desses desvios torcionais, consistindo no deslocamento lateral ou medial da tuberosidade tibial (TT), permitindo o reestabelecimento da posição da patela no sulco troclear do fêmur. Esta técnica é considerada indispensável por muitos autores, mesmo nos casos mais graves. A TTTT® (Tibial Tuberosity Transposition Tool) é técnica que permite o deslocamento da TT após osteotomia parcial e que não necessita de fixação da TT deslocada com banda de tensão. O presente trabalho tem por objetivo comparar os aspectos relacionados à execução, condições de aplicabilidade e incidência de complicações pós-operatórias da técnica tradicional de transposição e fixação da tuberosidade tibial, em relação à TTTT®.
\end{abstract}

PALAVRAS-CHAVE: Cão. Luxação de patela. Transposição da tuberosidade tibial.

\section{SUMMARY}

The patellar dislocation is a important casuistry among the orthopedic disorders affecting the dog's hindlimb. There's a consensus that patellar luxation is a clinical sign and not the disease, caused by underlying musculoskeletal deformities, which we can highlight the external or internal torsion of the proximal tibia. Tibial tuberosity Transposition (TTT) is the technique commonly performed to correct these torsional deviations, consisting on lateral or medial displacement of the tibial tuberosity (TT), allowing the restoration of the patella neutral path in the femoral trochlear groove. This technique is considered indispensable by many authors, even in the most severe cases. The TTTT® (Tibial Tuberosity Transposition Tool) provides a method that allows the displacement of tibial tuberosity, after partial osteotomy, that doesn't require fixation of the transposed tuberosity with tension band wire. The present paper aims to compare the aspects related to performance, applicability and incidence of postoperative complications of the established technique of transposition and attachment of tibial tuberosity in relation to TTTT®.

KEY-WORDS: Dog. Patellar luxation. Tibial tuberosity transposition.

\footnotetext{
${ }^{1}$ Médica Veterinária autônoma. Universidade Federal do Rio Grande do Sul. [E-mail: carlalcvet@ hotmail.com].

${ }^{2}$ Médico Veterinário autônomo. Mestre em Cirurgia de Pequenos Animais e especialista em Ortopedia de Pequenos Animais. Universidade Federal do Rio Grande do Sul.

${ }^{3}$ Professor adjunto do Departamento de Medicina Animal da Faculdade de Veterinária. Universidade Federal do Rio Grande do Sul.
} 


\section{INTRODUÇÃO}

A luxação patelar está entre as afecções ortopédicas mais comuns que acometem o joelho dos cães, sendo frequentemente diagnosticada em raças toy e miniatura, como Poodle, Yorkshire Terrier, Spitz Anão, Pinscher Miniatura e Shih Tzu (SOUZA et al., 2009). Também é observada em cães de grande porte, como São Bernardo, Chow-chow e Labrador Retriever (GIBBONS et al., 2006).

Esta afecção pode ser congênita ou traumática e, no que diz respeito à direção da luxação, pode ser classificada em medial, lateral ou bidirecional, sendo a luxação de patela medial congênita a mais frequentemente observada (HANS et al., 2016). A apresentação bilateral da luxação é mais comumente observada do que unilateral, de acordo com Stanke et al. (2014).

É consenso que a luxação patelar é sinal clínico causado por anormalidades musculoesqueléticas, que envolvem as estruturas do mecanismo extensor (BOJRAB, 2014), dentre as quais podemos destacar o arrasamento do sulco troclear, hipoplasia da crista troclear medial, torção interna ou externa da porção distal do fêmur e porção proximal da tíbia e coxa valga ou vara.

Os sinais clínicos relacionados à luxação patelar medial (LPM) variam de acordo com a classificação clínica do grau de luxação, conforme sistema descrito por Putnam e adaptado por Singleton (Tabela 1). Os graus de luxação estão correlacionados com a gravidade das anormalidades anatômicas (DECAMP et al., 2016).

O diagnóstico é baseado na história clínica, palpação do joelho afetado com o paciente em estação e em decúbito lateral e exame radiográfico com a realização de duas projeções ortogonais, para avaliação da presença e gravidade das deformidades esqueléticas, bem como de sinais de osteoartrose.

A patela luxa espontaneamente de forma intermitente à flexão do joelho, porém retorna
àn posição normal quando o joelho é estendido. Pode ser acompanhada por sinais de
claudicação indolor e saltitante. Pode ser observada rotação interna da tíbia entre 15 e
$20^{\circ}$ e abdução do tarso.
A patela permanece luxada, mesmo após extensão do joelho, retornando ao sulco
troclear apenas sob pressão manual. São observadas rotação interna acentuada da tíbia
entre $30^{\circ}$ e $60^{\circ}$ e curvatura em forma de S da porção distal do fêmur e proximal da
tíbia. O cão frequentemente utiliza o membro em posição semiflexionada, adotando
postura "agachada".
A patela não retorna ao sulco troclear, mesmo sob pressão manual. A tíbia sofre
rotação de $60^{\circ}$ para $90^{\circ}$, com relação ao plano sagital. Sem a correção do quadro no
início da vida do paciente, ocorrerá o desenvolvimento de deformidades ósseas e
ligamentosas graves, frequentemente irreparáveis.

A patela pode ser manualmente luxada, mas retorna à posição normal quando o joelho é estendido. Não há qualquer sinal de crepitação. Há o desenvolvimento de deformidades torcionais tibiais brandas (até $15^{\circ}$ ). Não há sinais clínicos, ou sua ocorrência é pouco frequente. posição normal quando o j claudicação indolor e saltitante. Pode ser observada rotação interna da tíbia entre 15 e A patela permanece luxada, mesmo após extensão do joelho, retornando ao sulco tíbia $\mathrm{O}$ cão frequentemente utiliza 0 membro em posição semiflexionada, adotando ligamentosas graves, frequentemente irreparáveis.

Tabela 1 - Sistema de classificação clínica do grau de luxação patelar medial descrito por Putnam e adaptado por Singleton. Fonte: DECAMP et al. (2016).

O manejo conservador da luxação patelar inclui a administração de anti-inflamatórios não-esteroidais e condroprotetores, permanecendo restrito aos casos de claudicação ocasional ou intermitente. Diversas técnicas cirúrgicas têm sido descritas para tratamento desta afecção (ARTHURS et al., 2006; SLATTER, 2007).

Este trabalho tem por objetivo descrever a transposição da tuberosidade tibial (TTT) e comparar os aspectos relacionados à execução, resposta pósoperatória de curto prazo, incidência de complicações pós-operatórias e limitações da técnica tradicional de fixação da tuberosidade tibial em relação à técnica recentemente desenvolvida e descrita por Petazzoni (2015), a TTTT®, no tratamento da luxação de patela em cães.

\section{MATERIAL E MÉTODOS}

Pesquisa bibliográfica foi utilizada para o desenvolvimento do presente trabalho. Inicialmente, revisão geral sobre os aspectos epidemiológicos, etiológicos, fisiopatológicos, clínicos, semiológicos e terapêuticos da luxação patelar canina foi realizada para situar o leitor dentro do tema proposto. Foi escolhida, especificamente, a técnica cirúrgica de transposição da tuberosidade tibial como escopo principal do trabalho, seguido da descrição de dois métodos distintos de execução da mesma, conforme literatura previamente publicada sobre o assunto. Buscou-se estabelecer comparação dos resultados obtidos por diferentes autores em estudos clínicos retrospectivos e prospectivos com a utilização desses métodos.

Essas informações foram obtidas por meio de compilações de dados contidas em livros de cirurgia veterinária e em artigos publicados em periódicos científicos, em formato físico ou eletrônico, devidamente referenciados. Como ferramenta virtual de pesquisa foi utilizado o Portal de Periódicos da CAPES (Coordenação de Aperfeiçoamento de Pessoal de Ensino Superior). 


\section{RESULTADOS E DISCUSSAO}

\section{Transposição da tuberosidade tibial: nova abordagem}

Historicamente, os planos de tratamento cirúrgico para a luxação patelar são baseados na classificação do grau clínico da luxação desenhado por Putnam e adaptado por Singleton (DECAMP et al., 2016; SLATTER, 2007). Diversos estudos têm considerado a TTT ponto-chave do tratamento cirúrgico da luxação patelar em cães e alguns têm demonstrado haver correlação entre a técnica e a redução da ocorrência de recidiva ou reluxação (ARTHURS et al., 2006). Essa classificação é baseada nos sinais clínicos (grau da luxação patelar) que não necessariamente refletem a magnitude das deformidades anatômicas subjacentes. O diagnóstico consiste em determinar onde e qual a magnitude da malformação que causa o deslocamento da patela.

A luxação de patela é secundária apenas às deformidades do fêmur nos planos frontal e transversal (varus ou valgus anormal e torção distal, interna ou externa) e às deformidades da tíbia no plano transversal (torção). De acordo com Petazzoni et al. (2015), valgus ou varus anormal da tíbia proximal e as deformidades no plano sagital do fêmur e tíbia não afetam o posicionamento da patela. Durante o crescimento, a tíbia proximal pode ser submetida à força rotacional (interna ou externa) anormal, sendo a torção externa mais frequente. É possível que a torção seja influenciada pela tensão que o quadríceps exerce sobre a tuberosidade tibial, através do tendão patelar e, neste caso, toda a tíbia proximal (incluindo o platô tibial e não apenas a crista) é submetida à torção em relação à porção distal. No osso imaturo, mais susceptível ao remodelamento, é possível que a torção não seja homogênea, estando a crista tibial mais rotada em relação ao centro da tíbia proximal (platô tibial). Se esta hipótese for verdadeira, a transposição da tuberosidade tibial não é a técnica corretiva mais apropriada, pois quando esta é reposicionada, o platô tibial permanece rotado, desviado obliquamente, no plano transversal (PETAZZONI, 2015).

A literatura relata taxas de sucesso elevadas com a utilização da TTT em casos mais graves - acima do grau II de luxação (torção tibial superior a $20^{\circ}$ ). É possível que a patela seja reduzida com o deslocamento da tuberosidade tibial em casos em que a torção da tíbia proximal é superior a $20^{\circ}$, pois as estruturas de suporte do joelho (ligamentos colaterais, cruzados e meniscos) permitem que a tíbia gire para se adaptar ao redirecionamento do trajeto patelar. $\mathrm{O}$ diagnóstico radiográfico (ou por tomografia computadorizada) torna-se necessário para determinar qual é a deformidade anatômica (ou as deformidades) subjacente(s) que causa(m) a luxação da patela. Exame radiográfico nas projeções mediolateral e craniocaudal devem ser obtidas do fêmur e da tíbia, individualmente. Para evitar interferências na determinação do ângulo de torção externa ou interna da $\mathrm{TT}$, relacionadas ao posicionamento radiográfico, convencionou-se que o córtex medial do osso calcâneo deve sobrepor o centro da cóclea tibial, conforme descrito por Petazzoni (2015). Para tanto, a extremidade distal da tíbia deve ser elevada, formando ângulo de $90^{\circ}$ com o feixe de radiação. A magnificação resultante deve ser levada em conta na mensuração do ângulo de torção. A posição da tuberosidade tibial no plano frontal e os respectivos ângulos de torção estão demonstrados na Figura 1.

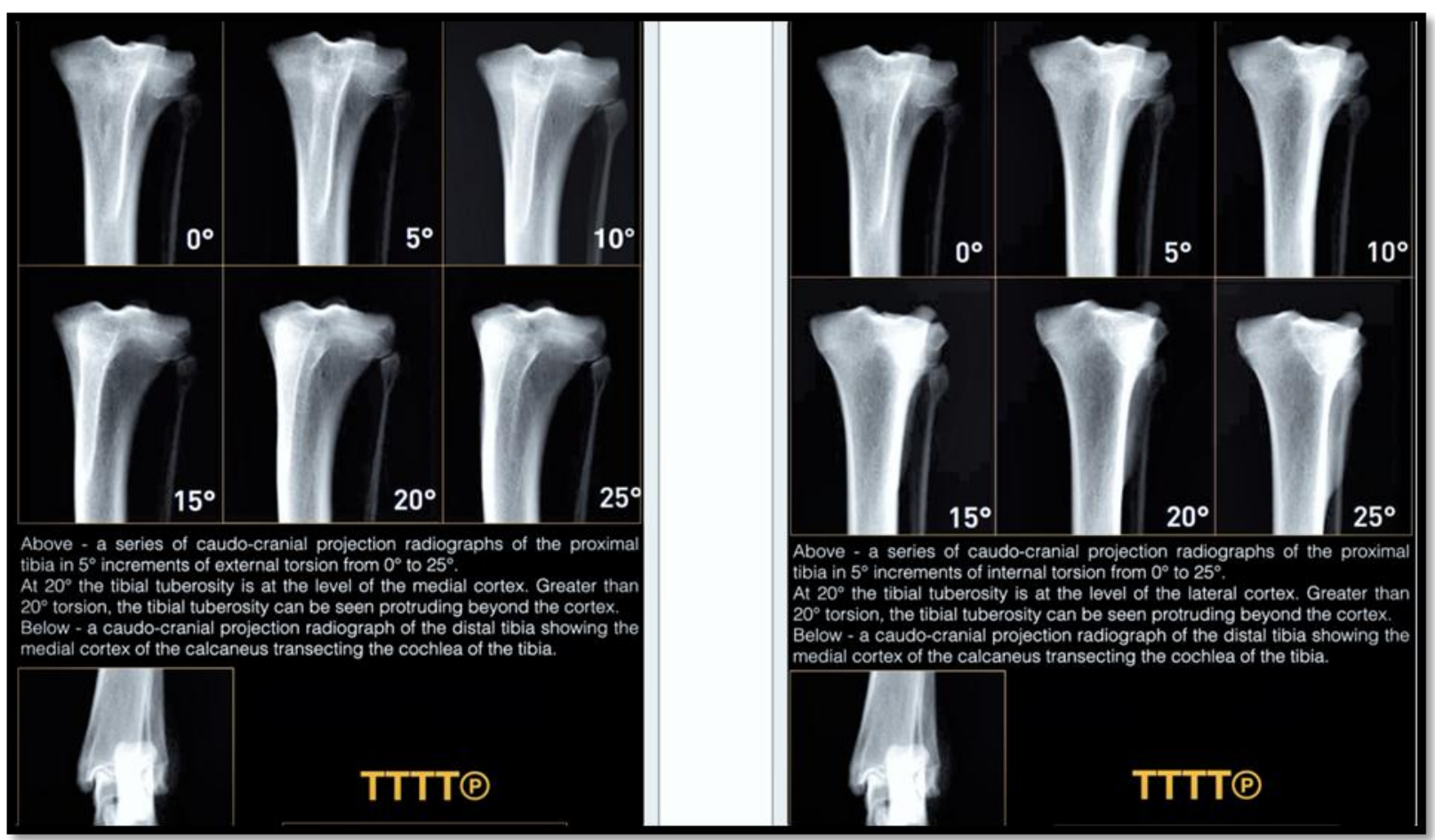

Figura 1 - Posição da tuberosidade tibial no plano frontal e os respectivos ângulos de torção externa (à esquerda) e interna (à direita). Fonte: PETAZZONI, 2015. 
Os graus de torção do fêmur distal são determinados pela avaliação da posição das fabelas em relação ao fêmur. Se a luxação for predominantemente secundária à torção tibial, o tratamento cirúrgico mediante realização da TTT pode proporcionar resultados satisfatórios, quando a torção é de até $20^{\circ}$ (torção máxima correspondente ao grau II da classificação de Singleton). Para os casos em que o ângulo de torção tibial seja superior a $20^{\circ}$, sugere-se a realização de osteotomia da tíbia proximal, como técnica cirúrgica corretiva, em substituição à TTT.

\section{Diferenças da TTTT® em relação à técnica tradicional}

Quando o desvio da tuberosidade tibial é a causa da luxação de patela, sua transposição para posição mais cranial leva ao realinhamento do mecanismo extensor. Dois métodos têm sido propostos para a TTT: osteotomia completa da TT em relação ao plano frontal, quando o osso e periósteo distais são completamente separados, permitindo o deslocamento lateral/medial e proximal/distal da TT; e osteotomia parcial, quando o osso e periósteo distais permanecem intactos. Nesta técnica, a crista tibial é rotacionada antes da fixação final, com o auxílio de pinos, o que melhora a estabilidade do fragmento osteotomizado, reduz o tempo cirúrgico e não requer a aplicação de banda de tensão. É indicada apenas nos casos em que a torção interna ou externa da TT é de, no máximo, $20^{\circ}$ (PETAZZONI, 2015).
A rotação aguda do fragmento pode levar à fratura da inserção distal do ligamento patelar, exigindo a aplicação de banda de tensão para evitar avulsão da TT, causada pela contração do músculo quadríceps. Decamp et al. (2016) descreveram variação da técnica de osteotomia total, em que uma borda óssea de 3 a $4 \mathrm{~mm}$ na tíbia proximal é deixada intacta e, a TT, fixada 5 a $8 \mathrm{~mm}$ distal a essa margem, para cães de pequeno porte (1,0 a 1,5 cm para cães de grande porte). Esse método pode ser utilizado para correção de patela alta, trazendo-a à sua posição anatômica, no terço proximal da tróclea femoral.

A TT é usualmente mantida na posição mediante fixação temporária do fragmento com fios de Kirschner ou utilização de pinça de redução ou garra óssea durante verificação do trajeto patelar antes da fixação definitiva. A fixação temporária com pinos pode fragilizar a tuberosidade, enquanto a pinça pode causar lesões aos tecidos moles adjacentes.

A TTTT $®$ é método que permite o deslocamento da TT após osteotomia parcial, sem a necessidade de fixação temporária da tuberosidade transposta e fixação definitiva com banda de tensão. A técnica de osteotomia parcial proposta por Petazzoni (2015), ilustrada na Figura 2, corresponde a $80 \%$ do comprimento da crista no aspecto medial e a $60 \%$ no aspecto lateral, para as luxações mediais de patela. A osteotomia é realizada mais caudalmente em relação à técnica tradicional, ao nível do Tubérculo de Gerdi, correspondendo a $30 \%$ do diâmetro da tíbia proximal.

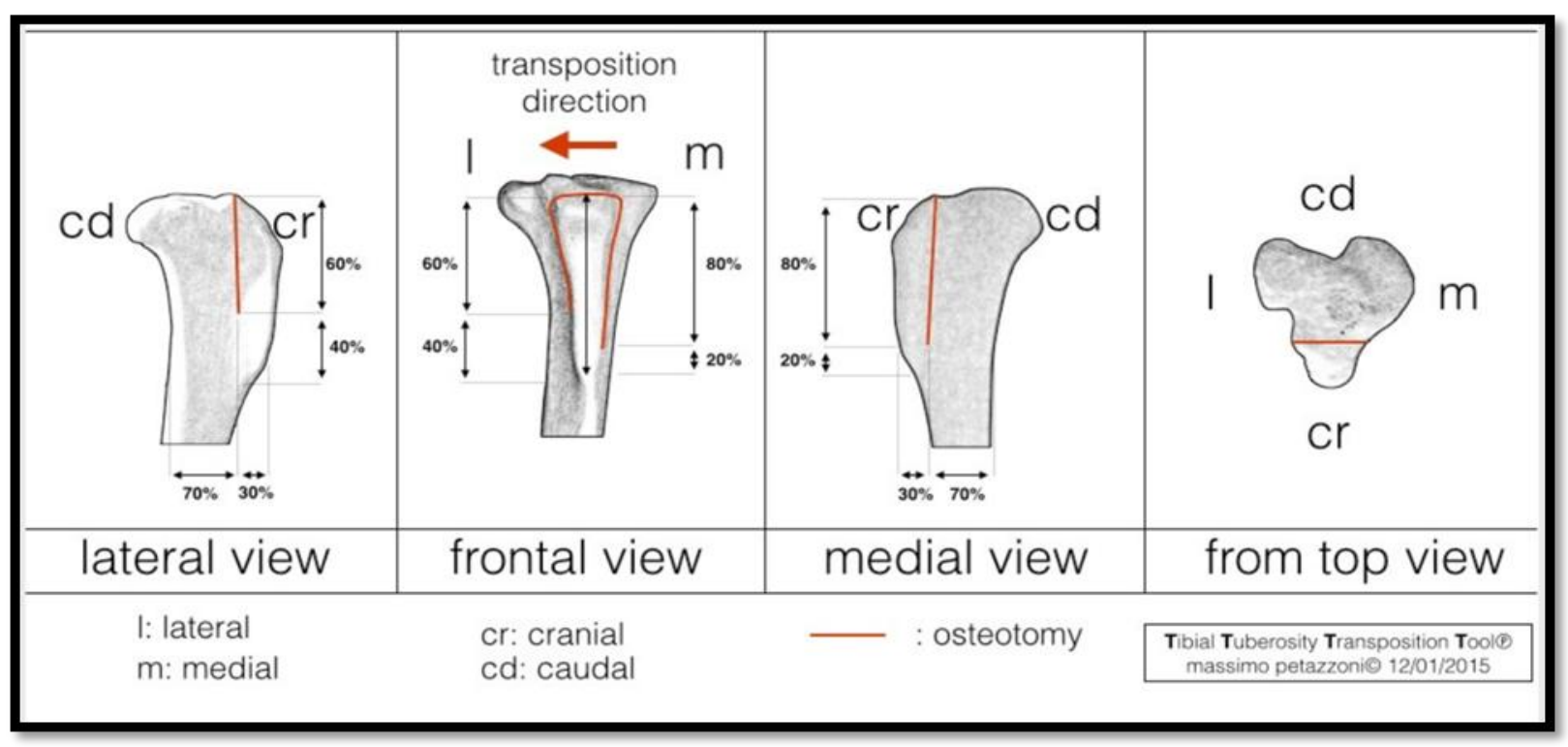

Figura 2 - Técnica de osteotomia parcial proposta por Petazzoni (2015), para a luxação de patela medial.

O deslocamento gradual da tuberosidade é obtido com a utilização de distrator ósseo (Figura 3), que permite a lateralização da TT, mantendo o fragmento na posição até a observação do trajeto neutro da patela no sulco troclear. Esse dispositivo é confeccionado com barra conectora rosqueada, bucha sulcada, chapa deslizante, duas porcas e dois pinos (lisos ou rosqueados), fixados craniocaudalmente e proximodistalmente nos aspectos medial e lateral da TT e dispostos de forma convergente.
O ângulo de inserção dos pinos corresponde ao ângulo de inclinação do platô tibial, em relação ao plano sagital e o ângulo entre os pinos, no plano transversal é de aproximadamente $70^{\circ}$. A porca é girada e a chapa desloca lateralmente a tuberosidade tibial, à velocidade de uma volta por minuto $(0,7 \mathrm{~mm})$. É recomendado um quarto de volta a cada 15 segundos. O deslocamento desejado em milímetros e o tempo necessário em minutos e segundos estão relacionados na Tabela 2. 


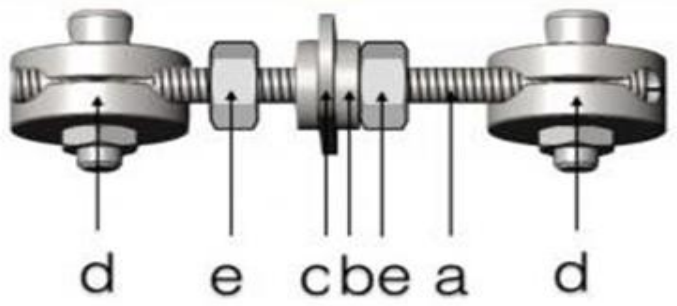

1) Assembled TTTTC: frontal view. TTTT(C) consists of one threaded rod (a), one grooved bushing (b) one sliding paddle (c), two clamps (d), two nuts (e)

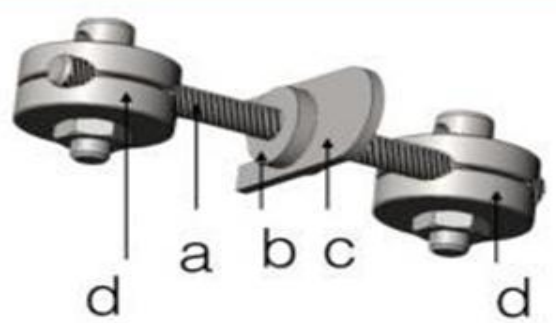

2) Assembled TITT(B); from bottom oblique frontal view. TITT(1) consists of one threaded rod (a). one grooved bushing (b), one sliding paddle (c), two clamps (d), two nuts (removed in this image to better show the grooved bushing and the sliding paddle)

Figura 3 - Ilustração esquemática do distrator ósseo utilizado na técnica TTTT®. Fonte: PETAZZONI, 2015.

\begin{tabular}{cccc}
\hline $\mathrm{A}$ & $\mathrm{B}$ & $\mathrm{C}$ & $\mathrm{D}$ \\
$\mathrm{mm}$ & voltas & minutos & 26 \\
1,0 & 1,4 & 1 & 9 \\
1,5 & 2,1 & 2 & 51 \\
2,0 & 2,9 & 2 & 34 \\
2,5 & 3,6 & 3 & 17 \\
3,0 & 4,3 & 4 & 0 \\
3,5 & 5,0 & 5 & 43 \\
4,0 & 5,7 & 5 & 26 \\
4,5 & 6,4 & 6 & 9 \\
5,0 & 7,1 & 7 & 26 \\
\hline
\end{tabular}

Tabela 2 - Relação entre o deslocamento da TT, necessário para o reestabelecimento do trajeto neutro da patela no sulco troclear e o tempo, de acordo com a técnica TTTT®. Fonte: PETAZZONI, 2015.

A taxa de deslocamento da tuberosidade influencia a capacidade do osso de se adaptar às forças deformantes aplicadas à porção distal da crista tibial. A TT é mantida na posição pela inserção de um pino liso ou rosqueado entre a tuberosidade e o córtex medial da tíbia osteotomizada (Figura 4), atuando como espaçador para prevenir o retorno da TT à posição original, causado pela memória elástica do osso. A distância entre o córtex medial da tíbia osteotomizada e a borda medial da TT, na altura da inserção do tendão patelar corresponde ao diâmetro do pino utilizado na fixação final. Após consolidação óssea completa (união radiográfica), o implante pode ser removido.

\section{Complicações e resposta clínica pós-operatória}

Em cães, a frequência de complicações pósoperatórias totais após tratamento cirúrgico da LPM varia de $18 \%$ a $43 \%$ (ARTHURS et al., 2006; GALLEGOS et al., 2016; STANKE et al., 2014;). O índice de complicações maiores relacionadas à TTT varia de $13 \%$ a $18 \%$ (ARTHURS et al., 2006). As complicações mais comuns são as associadas aos implantes (migração dos pinos, deiscência e formação de seroma no ponto de contato dos implantes com a pele e falha/quebra do implante), recorrência da luxação e avulsão da TT. Esta última nunca foi relatada, quando da utilização de pinos associada à banda de tensão. Fatores de risco para o desenvolvimento de complicações pósoperatórias incluem raças de grande porte e maior peso corporal. A avulsão da TT foi 11 vezes mais frequente com a utilização de fio de Kirschner único em relação à fixação com dois fios de Kirschner. A despeito do número de pinos utilizados, o direcionamento caudodistal foi correlacionado com risco maior de avulsão da TT. Recorrências são relatadas na ordem de $8 \%$ a $36 \%$ dos casos (ARTHURS et al., 2006; GALLEGOS et al., 2016; GIBBONS et al., 2006). Casos de luxação lateral de patela, após correção cirúrgica da LPM, causada pelo deslocamento lateral excessivo da TT, também têm sido descritos. 


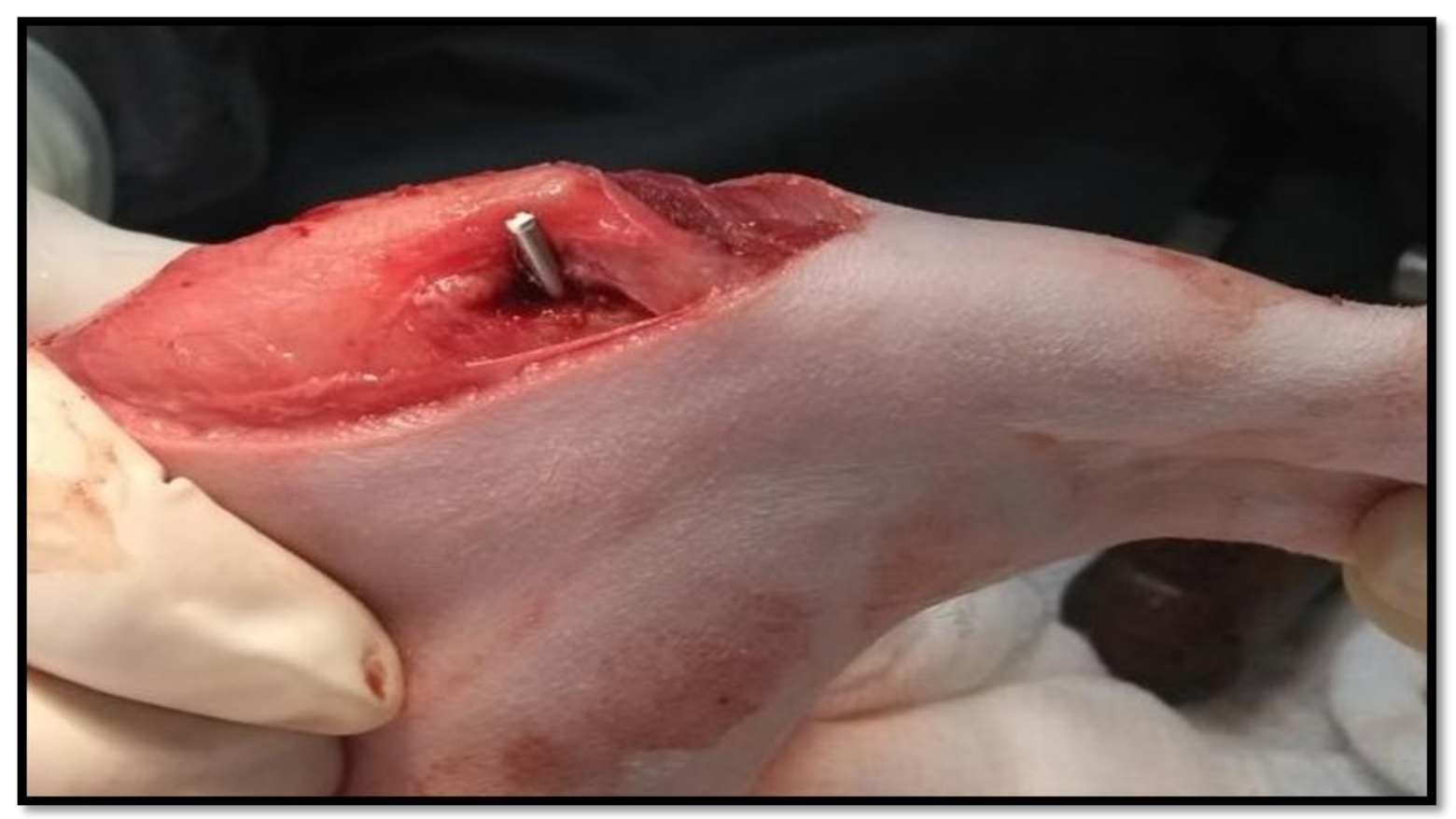

Figura 4 - Fixação final da TT obtida pela inserção de um pino entre a tuberosidade e o córtex medial da tíbia osteotomizada.

Stanke et al. (2014) avaliaram retrospectivamente os fatores de risco para o desenvolvimento de complicações totais e maiores após TTT em 113 cães com luxação medial de patela. Das variáveis relacionadas ao paciente, a idade maior no momento da cirurgia foi correlacionada com risco menor de desenvolvimento de complicações totais (com um decréscimo de $20 \%$ na probabilidade de ter qualquer complicação a cada ano adicional). Raças de grande porte tiveram 5,5 vezes mais chance de desenvolver complicações maiores do que cães de pequeno porte. Em relação à variável peso, a cada incremento de $5 \mathrm{~kg}$, a probabilidade de complicações maiores aumentou em 1,3 vezes, o que pode ser explicado pelas forças maiores aplicadas sobre os implantes, com risco maior de falha ou quebra antes da consolidação completa da região osteotomizada, necessitando de reintervenção cirúrgica. A correlação entre peso corporal e o desenvolvimento de complicações também foi demonstrada por Gibbons et al (2006), com percentual de complicações totais na ordem de $29 \%$. Dos métodos de fixação avaliados (um, dois ou três pinos lisos sem banda de tensão, um ou dois pinos rosqueados sem banda de tensão, um parafuso compressivo associado a um ou dois pinos e um ou dois pinos associados à banda de tensão), apenas a fixação com parafuso compressivo foi correlacionada com risco maior de complicações pós-operatórias.

Em estudo prospectivo, Petazzoni (2015) avaliou as respostas clínica e radiográfica de curto prazo da técnica TTTT® em 19 cães com luxação patelar medial de graus I e II, uni ou bilateral. Os cães que apresentavam torção do fêmur distal, além da torção tibial proximal, não foram incluídos. Foi realizada a ressecção em cunha da tróclea femoral, além da TTTT® em 29 joelhos. A claudicação era menos evidente com duas semanas de pós-operatório e, com oito semanas, a recuperação funcional do membro era completa em todos os pacientes. Não foi observada recorrência da luxação nos joelhos tratados. A consolidação completa da região osteotomizada foi observada com oito semanas, mediante avaliação radiográfica, em todos os cães. Foi relatada apenas uma complicação intraoperatória e uma complicação menor em dois cães, sem ocorrência de complicações maiores.

\section{CONCLUSÕES}

A transposição da tuberosidade tibial é uma das técnicas consideradas de eleição pela maioria dos autores, estando incluída nos planos de tratamento cirúrgico para correção da luxação patelar canina, mesmo nos casos mais graves. Entretanto, a nova metodologia proposta para execução da técnica que é apresentada neste trabalho segue abordagem distinta da tradicionalmente empregada. Segundo ela, a forma como as deformidades anatômicas que causam a luxação da patela ocorrem deve ser criteriosamente avaliada e sugere que a TTT nem sempre é a técnica corretiva mais adequada, ficando restrita apenas aos graus I e II da classificação de Putnam. A TTTT® representa alternativa à técnica tradicional, com menor incidência de complicações pós-operatórias, de acordo com alguns estudos já realizados. Entretanto, estudos adicionais são necessários para avaliar a resposta pósoperatória de longo prazo nesses pacientes e em cães de grande porte.

\section{AGRADECIMENTOS}

Agradeço imensamente ao meu querido mestre e amigo, Leandro Haczkiewicz Gaiga, pela sugestão do tema do presente trabalho e por todo aprendizado adquirido durante esses quatro anos de estágio, 
relacionado não somente à ortopedia, mas à cirurgia geral também. Agradeço também ao meu orientador, Márcio Poletto Ferreira, pelo aceite de orientação e pelas sugestões recebidas no decorrer da realização deste trabalho.

\section{REFERÊNCIAS}

ARTHURS, G.I.; LANGLEY-HOBBS, S.J. Complications associated with corrective surgery for patellar luxation in 109 dogs. Veterinary Surgery. v.35, n.6, p.559-66, 2006.

BOJRAB, M.J. Mecanismos das Doenças em Cirurgia de Pequenos Animais. 3.ed. São Paulo: Roca, 2014. cap.106, p.856-63.

DECAMP, C.E.; JOHNSTON, S.A.; DÉJARDIN, L.M.; SCHAEFER, S.L. Brinker, Piermattei and Flo's Handbook of Small Animal Orthopedics and Fracture Repair. 5.ed. Saint Louis: Elsevier, 2016. cap.18, p.597-616.

GALLEGOS, J.; UNIS, M.; ROUSH, J.K.; AGULIAN, L. Postoperative complications and short-term outcome following single-session bilateral corrective surgery for medial patellar luxation in dogs weighing < $15 \mathrm{~kg}$ : 50 cases (2009-2014). Veterinary Surgery. v.45, p.88792, 2016.

GIBBONS, S.E.; MACIAS, C.; TONZING, M.A.; PINCHBECK, G.L.; McKEE, W.M. Patellar luxation in 70 large breed dogs. Journal of Small Animal Practice. v.47, p.3-9, 2006.
HANS, E.C.; KERWIN, S.C.; ELLIOT, A.C.; BUTLER, R.; SAUNDERS, W.B.; HULSE, D.A. Outcome following surgical correction of grade 4 medial patellar luxation in dogs: 47 stifles (20012012). Journal of American Animal Hospital Association. v.52, n.3, p.162-9, 2016.

PETAZZONI, M. Tibial Tuberosity Transposition Tool TTTT® and Technique Manual. 2015. 65p. Disponível em: http://itunes.apple.com/us/book/tibialtuberosity-transposition/id970657931?s=1\&mt=11.

SLATTER, D. Manual de Cirurgia de Pequenos Animais. 3.ed. Barueri: Manole, 2007. v.2, cap.147, p.2122-26.

SOUZA, M.M.D.; RAHAL, S.C.; OTONI, C.C.; MORTARI, A.C.; LORENA, S.E.R.S. Luxação de patela em cães: estudo retrospectivo. Arquivo Brasileiro de Medicina Veterinária e Zootecnia. v.61, n.2, p.523-26, 2009.

STANKE, N.J.; STEPHENSON, N.; HAYASHI, K. Retrospective risk factor assessment for complication following tibial tuberosity transposition in 137 canine stifles with medial patellar luxation. Canadian Veterinary Journal. v.55, p.349-56, abr. 2014. 